MATEC Web of Conferences 22,02018 (2015)

DOI: $10.1051 /$ matec conf/ 20152202018

(C) Owned by the authors, published by EDP Sciences, 2015

\title{
Efficiency Analysis of Wireless Power Transmission for Portable Elec- tronics
}

\author{
Xingping Xu, Chuanxiang Yu, Yuxin Zhang, Man Zhang \& Haotian $\mathrm{Hu}$ \\ School of Electrical Engineering, Chongqing University, Chongqing, China
}

\begin{abstract}
With the portable electronics becoming more and more popular, a model of wireless power transmission for general portable electronics was presented based on the principles of magnetic resonance transmission. And that transferring the model to an equivalent circuit was possible to calculate. The characteristics of the system and the optimal conditions were analyzed, and the influence of the transmission efficiency for the Coupling factor and the Quality factor was discussed. The theory analysis and simulation in MATLAB show that the performance of the system can be optimized by adjusting the coupling factor between the transmitter coupling coils. Furthermore, the feasibility of this theory was proven by experiments. Thus, this paper provides a useful reference to improve the wireless power transmission for portable electronics.
\end{abstract}

Keywords: portable electronics; wireless power transmission; magnetic coupling resonance; efficiency

\section{INTRODUCTION}

In November 2006, an assistant professor of department of physics in MIT, Marin Soljacic, first proposed magnetic coupling resonant WPT technology on the BBS in AIP industrial physics. And in June 2007, using the technology, he successfully lit a 60 -watt light bulb in the distance of 2.13 meters. Efficiency is as high as $96 \%$ at a distance of $75 \mathrm{~cm}$. The news seems to ignite the enthusiasm of scientists. With the portable electronic products (such as TVs, laptops, cell phones, and so on) were more and more popular, people's lives become more convenient. At the same time, the traditional power supply of these devices cause a lot of inconvenience to people, such as the limited power supply, power transmission and other security risks. In order to effectively address the shortcomings of traditional wired power supply in recent years, the wireless power transmission (WPT) has attracted much attention of researchers at home and abroad [1]. Currently, the wireless power transmission mode including: the resonance magnetic coupling type, the electromagnetic induction type, and the electromagnetic radiation type. Among them, the resonant magnetic coupling technique was the use of non-radiative electromagnetic near-field region achieving energy transmission. Compared with the other two types, it has advantages such as low difficulty design, high transmission efficiency, long transmission distance, no radiation and high security features. So this type of wireless power transmission system has received more attentions and researches [2-3].

In order to obtain a stable system performance, the transmitter and the receiver in the systems usually use the same parameters, symmetrical resonator to achieve excellent performance [4-5]. However, it owns a very narrow range of applications of the study because of the actual transmission distance and equipment size. In the reference [6], the author presented the magnetic coupling of the resonance system made by different size and asymmetric resonators without the theoretical point of view of the working mechanism, and optimization of system performance; the references [7-8] are presented to adjust the distance between the transmitter and receiver to achieve impedance matching system, thereby improve the transmission efficiency. However, four coils in the system separated from each other need to keep the same distance, besides, the load impedance should also remain the same. So it was not for the actual electronic products. Reference [9] shows that the professor TC Beth had use the wireless power transmission in electric automobile, studying the receive coil resonance frequency and system frequency which were caused by mismatch problem. Impedance matching was put forward to adjust the coil resonance frequency. Besides, the electromagnetic analysis and test was carried on to conclude a parameter design method. Scholars such as Seung-Hwan LEE proposed an equivalent circuit model of the magnetic coupling resonant WPT system in the reference [10]. And the finite element analysis shows that the circuit model is an effective model which including the influence of skin effect and proximity effect based on a high frequency system. All above has helped to promote the progress of wireless power transmission.

This paper presents a suitable portable electronic products magnetically coupled resonant coupling wireless power transmission system model to fill up the deficiency mentioned earlier [11-12]. The transmission characteristics of the system were analyzed, the expressions of the transmission efficiency were derived, the system performance was optimized, and the impact of the quality factor and the coupling factor for transmission efficiency was discussed. Finally, the 
optimum conditions in the system were given. The Matlab simulation shows that when the coil Q factor is determined, the system transmission efficiency optimization can be realized by adjusting the coupling factor between the transmitter coils.

\section{SYSTEM MODEL AND CIRCUIT ANALYSIS}

The model of wireless power transmission system for portable electronic products was shown in Figure 1. There were four components of the system consist of the drive coil (Drive Coil), the transmit coil (TX Coil), the receiving coil (RX Coil) and the load coil (Load Coil). Transmitting and receiving coils were called resonator. When energy was transmitted, they were in a resonance state. The resonant frequency and the natural frequency of the coil were the same. As shown in Figure 1 , the transmitting terminal was the same with the traditional four resonant wireless power transmitting, namely the driving coil was formed as the single-turn coil, and the multi-turn transmitter coil was made of axial spiral coil. But the receiving terminal of the receiving coil and loading coil in a planar spiral were fixed together and they are not the same size with the transmitter coil [13-14]. Thus, the coil size is in accordance with the size of the electrical equipment.

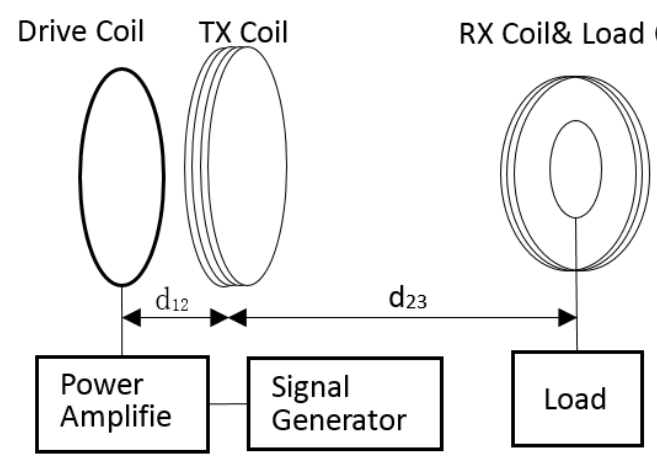

Figure 1. Model of wireless power transmission that suitable for portable electronics

To simplify the analysis, only four coils energy transfer were analyzed [15]. In accordance with the principle of magnetic coupling and the resonance circuit theory, each of coils may be equivalent to RLC circuit system. So it is obvious that we can obtain the equivalent circuit model shown in Figure 2. In the Figure 2, AC was a high-frequency signal source, L1, L2, L3 and L4 were the equivalent inductance of the four coils; RL was the load resistance; R1, R2, R3 and R4 were loss resistance of the coils; C1, C2, C3 and $\mathrm{C} 4$ were the sum of a parasitic capacitance and tuned compensation capacitor; M12, M23 and M34 were the mutual inductance between adjacent coils. Since no cross coupling between the adjacent, weak, low-impact coils, the cross mutual inductance M13, M14 and M24 were not considered in the process of analysis.

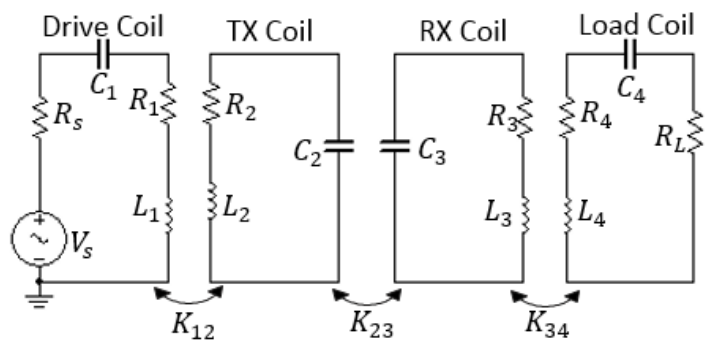

Figure 2. Equivalent circuit of wireless power transmission

In Figure 2, K12 was presented as the coupling factors between the Drive coil and the TX coil, K23 was presented as the coupling factors between the TX coil and the RX coil, K34 was presented as the coupling factors between the RX coil and the Load coil. They were mainly determined by coil inductance, coil turns and the distance among coils.

Assume $\omega$ was the angular frequency in the system. By Kirchhoff's voltage law (KVL), we can obtain that[16]:

$\left[\begin{array}{c}i_{1} \\ i_{2} \\ i_{3} \\ i_{4}\end{array}\right]=\left[\begin{array}{llll}Z_{11} & Z_{12} & Z_{13} & Z_{14} \\ Z_{21} & Z_{22} & Z_{23} & Z_{24} \\ Z_{31} & Z_{32} & Z_{33} & Z_{34} \\ Z_{41} & Z_{42} & Z_{43} & Z_{44}\end{array}\right]^{-}\left[\begin{array}{c}V S \\ 0 \\ 0 \\ 0\end{array}\right]$

Wherein:

$Z_{m n}=Z_{n m}=\left\{\begin{array}{c}R_{n}+j \omega L_{n}+\frac{1}{j \omega C_{n}}(m=n) \\ j \omega M_{m n}(m \neq n), M_{m n}=k_{m n} \sqrt{L_{m} L_{n}}\end{array}\right.$

Wherein $\mathrm{n}, \mathrm{m}$ was resonant coil numerals, $\mathrm{n}, \mathrm{m} \in$ $\{1,2,3,4\} ; R_{n}$ was presented as the $n$-th coil of equivalent resistance; $\mathrm{L}_{\mathrm{n}}$ was presented as the $\mathrm{n}$-th coil equivalent inductance; $C_{n}$ was the $n$-th tuning capacitance value; $\mathrm{M}_{\mathrm{mn}}$ and $\mathrm{k}_{\mathrm{mn}}$ were respectively the mutual inductance and the coupling coefficient between the $\mathrm{m}$-th coil and the $\mathrm{n}$-th coil. When the system was in steady-state operation, it was shown as follows:

$$
\begin{aligned}
& \omega=\frac{1}{\sqrt{L_{m} C_{n}}} n \in\{1,2,3,4\} \\
& j \omega L_{n}+\frac{1}{j \omega C_{n}}=0(m=n, n=\{1,2,3,4\})
\end{aligned}
$$

Ignore the cross-coupling factor k14, k13, k24, the formula (1) can be simplified as follows:

$$
\left[\begin{array}{c}
i_{1} \\
i_{2} \\
i_{3} \\
i_{4}
\end{array}\right]=\left[\begin{array}{cccc}
R_{1} & j \omega M_{12} & 0 & 0 \\
j \omega M_{12} & R_{2} & -j \omega M_{23} & 0 \\
0 & -j \omega M_{23} & R_{3} & j \omega M_{34} \\
0 & 0 & j \omega M_{34} & R_{4}
\end{array}\right]^{-1}\left[\begin{array}{c}
V_{s} \\
0 \\
0 \\
0
\end{array}\right] \text { (5) }
$$


Taking the quality factor $Q_{n}=\frac{\omega L_{n}}{R_{n}}$ and equation into the equation (5), it was shown as follows:

$$
\left\{\begin{array}{c}
i_{1}=\frac{\left(1+k_{23}^{2} Q_{2} Q_{3}+k_{34}^{2} Q_{3} Q_{4}\right) V_{s}}{R_{1}\left[\left(1+k_{12}^{2} Q_{1} Q_{2}\right)\left(1+k_{34}^{2} Q_{3} Q_{4}\right)+k_{23}^{2} Q_{2} Q_{3}\right]} \\
i_{2}=\frac{-k_{12} \sqrt{Q_{1} Q_{2}}\left(1+k_{34}^{2} Q_{3} Q_{4}\right) V_{s}}{\sqrt{R_{1} R_{2}}\left[\left(1+k_{12}^{2} Q_{1} Q_{2}\right)\left(1+k_{34}^{2} Q_{3} Q_{4}\right)+k_{23}^{2} Q_{2} Q_{3}\right]} \\
i_{3}=\frac{-k_{12} k_{23} \sqrt{Q_{1} Q_{2}} \sqrt{Q_{2} Q_{3}} V_{s}}{\sqrt{R_{1} R_{3}}\left[\left(1+k_{12}^{2} Q_{1} Q_{2}\right)\left(1+k_{34}^{2} Q_{3} Q_{4}\right)+k_{23}^{2} Q_{2} Q_{3}\right]} \\
i_{4}=\frac{-k_{12} k_{23} k_{34} \sqrt{Q_{1} Q_{2}} \sqrt{Q_{2} Q_{3}} \sqrt{Q_{3} Q_{4}} V_{s}}{\sqrt{R_{1} R_{4}}\left[\left(1+k_{12}^{2} Q_{1} Q_{2}\right)\left(1+k_{34}^{2} Q_{3} Q_{4}\right)+k_{23}^{2} Q_{2} Q_{3}\right]}
\end{array}\right.
$$

Expression (7) of transmission efficiency in the system was obtained by equation (6). In addition, the loading coil was usually a single coil, and R4 was much less than L4. It was possible for formula (7) to do approximation as shown in formula (8):

$$
\begin{aligned}
& \eta=\frac{1}{\left[1+\mathrm{k}_{23}^{2} \mathrm{Q}_{2} \mathrm{Q}_{3}+\mathrm{k}_{34}^{2} \mathrm{Q}_{3} \mathrm{Q}_{4}\right]} \times \\
& \frac{\left(\mathrm{k}_{12}^{2} \mathrm{Q}_{1} \mathrm{Q}_{2}\right)\left(\mathrm{k}_{23}^{2} \mathrm{Q}_{2} \mathrm{Q}_{3}\right)\left(\mathrm{k}_{34}^{2} \mathrm{Q}_{3} \mathrm{Q}_{4}\right)}{\left[\left(1+\mathrm{k}_{12}^{2} \mathrm{Q}_{1} \mathrm{Q}_{2}\right)\left(1+\mathrm{k}_{34}^{2} \mathrm{Q}_{3} \mathrm{Q}_{4}\right)+\mathrm{k}_{23}^{2} \mathrm{Q}_{2} \mathrm{Q}_{3}\right]} \\
& \eta=\frac{i_{4}^{2} R_{L}}{U_{s} i_{1}}=\frac{R_{L}}{R_{L}+R_{4}} \times \frac{1}{\left[1+\mathrm{k}_{23}^{2} \mathrm{Q}_{2} \mathrm{Q}_{3}+\mathrm{k}_{34}^{2} \mathrm{Q}_{3} \mathrm{Q}_{4}\right]} \times \\
& \frac{\left(\mathrm{k}_{12}^{2} \mathrm{Q}_{1} \mathrm{Q}_{2}\right)\left(\mathrm{k}_{23}^{2} \mathrm{Q}_{2} \mathrm{Q}_{3}\right)\left(\mathrm{k}_{34}^{2} \mathrm{Q}_{3} \mathrm{Q}_{4}\right)}{\left[\left(1+\mathrm{k}_{12}^{2} \mathrm{Q}_{1} \mathrm{Q}_{2}\right)\left(1+\mathrm{k}_{34}^{2} \mathrm{Q}_{3} \mathrm{Q}_{4}\right)+\mathrm{k}_{23}^{2} \mathrm{Q}_{2} \mathrm{Q}_{3}\right]}
\end{aligned}
$$

\section{PERFORMANCE ANALYSIS AND OPTIMI- ZATION OF SYSTEM}

According to the formula (8), it is shown that when the load was determined, the energy transfer efficiency was determined by the coupling factor and the quality factor. In order to further study the transmission efficiency of the optimized conditions, we respectively analyzed the transmission efficiency with the influence of the coupling factor and the quality factor.

\subsection{Impacts on the efficiency and optimization of the quality factor}

Figure 2 shows that the drive coil is connected to the power supply and the load coil is connected to the load. The quality factor Q1, Q4 was very small due to RS and RL. In the practical application, load coils were limited by size of the apparatus, which makes Q4, the size level, be generally less than 1; the quality factor Q2, Q3 was relatively independent, the self-resistors R2 and R3 were small, so Q2, Q3 was usually much greater than Q1, Q4. In addition, when the system was working properly, the transmitter and the receiver were in a state of strong coupling, and the coupling factor meets $\mathrm{k} 12, \mathrm{k} 34>>\mathrm{k} 23$. This time, the formula (8) can do the following approximations:

$\left(1+k_{12} Q_{1} Q_{2}\right)\left(1+k_{34}^{2} Q_{3} Q_{4}\right) \approx\left(k_{12}^{2} Q_{1} Q_{2}\right)\left(k_{34}^{2} Q_{3} Q_{4}\right)(9)$

$\left(1+\mathrm{k}_{12}^{2} \mathrm{Q}_{1} \mathrm{Q}_{2}\right)\left(1+\mathrm{k}_{34}^{2} \mathrm{Q}_{3} \mathrm{Q}_{4}\right)+\mathrm{k}_{23}^{2} \mathrm{Q}_{2} \mathrm{Q}_{3}$

$\approx\left(\mathrm{k}_{12}^{2} \mathrm{Q}_{1} \mathrm{Q}_{2}\right)\left(\mathrm{k}_{34}^{2} \mathrm{Q}_{3} \mathrm{Q}_{4}\right)$

$1+\mathrm{k}_{23}^{2} \mathrm{Q}_{2} \mathrm{Q}_{3}+\mathrm{k}_{34}^{2} \mathrm{Q}_{3} \mathrm{Q}_{4} \approx 1+\mathrm{k}_{23}^{2} \mathrm{Q}_{2} \mathrm{Q}_{3}$

At last, the equation (8) can be approximated as follows:

$$
\eta \approx \frac{k_{23}^{2} Q_{2} Q_{3}}{1+k_{23}^{2} Q_{2} Q_{3}}
$$

From the equation (12), we can know that the transmission efficiency was proportional to formula $k_{23}^{2} Q_{2} Q_{3}$. So, when $\mathrm{k} 23$ was determined, the transmission efficiency was only relied on the value of Q2, Q3. Since that:

$k_{m n} \approx \frac{\mu_{0} \pi N_{m} N_{n} r_{m}^{2} r_{n}^{2}}{2 \sqrt{L_{m} L_{n}} d_{m n}^{3}}$

Wherein: $\mu 0$ was the permeability of vacuum; $\mathrm{Nm}$, $\mathrm{Nn}$ was the circle number of coil $\mathrm{m}, \mathrm{n}$; rm, rn was the radius of coil $\mathrm{m}, \mathrm{n}$; dmn was the distance of coil $\mathrm{m}, \mathrm{n}$; $\mathrm{Lm}, \mathrm{Ln}$ was the inductance value of coil $\mathrm{m}, \mathrm{n}$ and $\mathrm{m}, \mathrm{n}$ $\in\{1,2,3,4\}$.

It comes to a conclusion from formula (13) that when the distance $\mathrm{d} 23$ was determined in practical application, we can improve the efficiency of transmission through changing the parameter of coil to get high transmission efficiency.

To further illustrate the impact of the quality factor $\mathrm{Q}$ of the transmission efficiency, we make $\mathrm{Vs}=15 \mathrm{~V}$, $\omega=7.53106 \mathrm{rad} / \mathrm{s}, \mathrm{R} 1=20, \mathrm{Q} 2=200, \mathrm{Q} 3=200, \mathrm{k} 12=$ $0.2, \mathrm{k} 23=0.5, \mathrm{k} 34=0.5$, and then, we obtain the relation curve through formula (8) shown in Figure 3. Next step, we make Vs $=15 \mathrm{~V}, \omega=7.53106 \mathrm{rad} / \mathrm{s}, \mathrm{R} 1$ $=20, \mathrm{Q} 1=0.8, \mathrm{Q} 4=0.8, \mathrm{k} 12=0.2, \mathrm{k} 23=0.5, \mathrm{k} 34=$ 0.5 , and then, we obtain the relation curve through formula (8) as shown in Figure 4.

As shown in Figure 3, the efficiency affected by quality factor Q1 was very small. With the change of Q4, there will be a peak efficiency. However, when Q4 was between the value of 11 and 20, it changed little. It can be seen from Figure 4 that the transmission efficiency is obviously increased with the change of quality factor Q2, Q3. So, while in the actual design of wireless power transmission system, the resonance frequency meets the requirements under the premise, 
the bigger value of quality factor Q2 and Q3, the better. This was much of value in the following analysis.

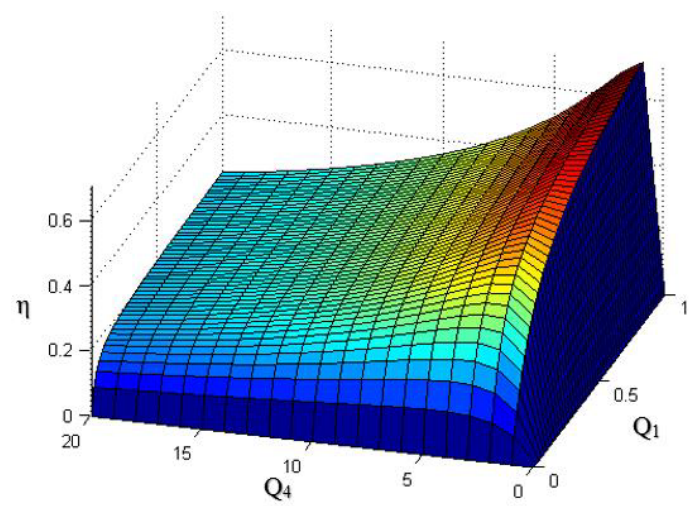

Figure 3. Relation curve between Q1, Q4 and $\eta$

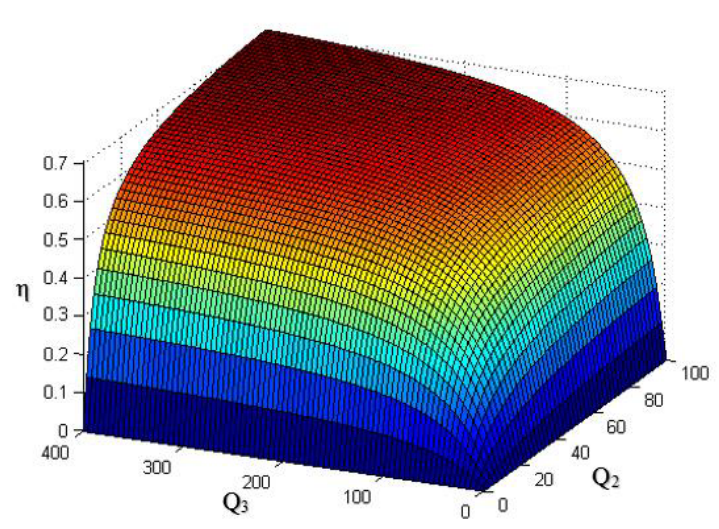

Figure 4. Relation curve of Q2, Q3 and $\eta$

\subsection{Impacts on the efficiency and optimization of coupling factor}

In practical applications, when the wireless power transmission system design was completed, the quality factor Q1 to Q4 were the fixed value. In addition, the receiving coil and loading coil secured together in this wireless power transmission system that we study, so k34 was also a fixed value. Therefore, the transmission efficiency was only influenced by k12, k23 indicated in the formula (8).

Take the derivative of formula (8) by $\mathrm{k} 23$ to obtain the maximum transmission efficiency. Then $\mathrm{k} 12$ and k23 need to meet the formula (14) shown as follows:

$$
k_{12}=\sqrt{\frac{1}{Q_{1} Q_{2}}} \cdot \sqrt{\left(\frac{k_{23}^{2} Q_{2} Q_{3}}{1+k_{34}^{2} Q_{3} Q_{4}}\right)^{2}-1}
$$

It is obviously seen from the formula (13) and (14) that when the efficiency is declined with the change of the equipment distance $\mathrm{d} 23$ and direction in practice application, we can adjust the distance $\mathrm{d} 12$ in the transmitting terminal to optimize the transmission efficiency.

\section{SIMULATION ANALYSIS AND EXPERIMENT VERIFICATION}

\subsection{Simulation analysis}

In order to verify the optimization analysis mentioned earlier, the simulation analysis by Pspice, the wireless power transmission system model, was done. Simulation circuit was shown in Figure 5. The simulation system was based on SPICE software.

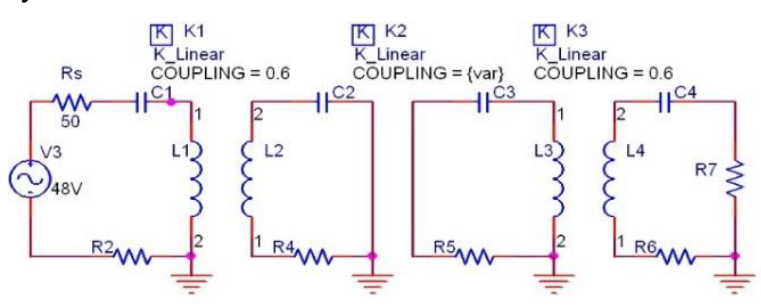

Figure 5. Simulation model of magnetically coupled resonant wireless power transmission system

Change the value of inductance, capacitance and resistance in the circuit to obtain the resonant state. At the same time, difference quality factors $\mathrm{Q}$ were obtained, which was the key point. Then, different value with different efficiency is shown in Table 1.

Table 1. Results of simulations $(\mathrm{k} 12=0.8, \mathrm{k} 23=0.3, \mathrm{k} 34=0.8$, $\mathrm{RL}=50 \Omega$ )

\begin{tabular}{|l|l|l|l|l|l|}
\hline$Q_{1}$ & $Q_{2}$ & $Q_{3}$ & $Q_{4}$ & /MHz & $\eta / \%$ \\
\hline 0.63 & 317 & 338 & 0.32 & 1.01 & 35 \\
\hline 0.63 & 525 & 338 & 0.32 & 1.01 & 52 \\
\hline 0.63 & 317 & 226 & 0.32 & 1.01 & 25 \\
\hline 0.63 & 317 & 226 & 0.23 & 1.01 & 30 \\
\hline 0.63 & 525 & 525 & 0.32 & 1.01 & 65 \\
\hline 0.63 & 317 & 338 & 0.32 & 1.01 & 38 \\
\hline 0.63 & 525 & 226 & 0.32 & 1.01 & 62 \\
\hline
\end{tabular}

We can see from Table 1 that the transmission efficiency changing with the quality factor $\mathrm{Q}$ was satisfied well with the theory analysis mentioned earlier. As shown in Figure 6, when Q1 to Q4 were determined and k23 was changeable, we obtain the curve of the transmission efficiency. In order to facilitate comparative analysis, we measured the corresponding transmission efficiency when the coupling factors were changed and fixed.

As shown in Figure 6, when k12 was a fixed value and $\mathrm{k} 23$ was increased to a certain value, the transmis- 
sion efficiency will be rapidly decreased. However, the transmission efficiency will be continued to increase through changing $\mathrm{k} 12$. This was because there will be a frequency splitting increased with $\mathrm{k} 23$ when $\mathrm{k} 12$ was a fixed value detuning the system and leading to a sharp decline in transmission efficiency. If changing $\mathrm{k} 12$ on the system is to realize the real-time adjustment, then the frequency splitting phenomenon does not occur, and it can effectively improve the transmission efficiency. Conclusion was consistent with the theoretical analysis. In practical applications, it can effectively increase the transmission distance and the transmission efficiency of energy by manual adjustment or automatic adjustment of the distance between the transmitter coils to find the point of maximum efficiency.

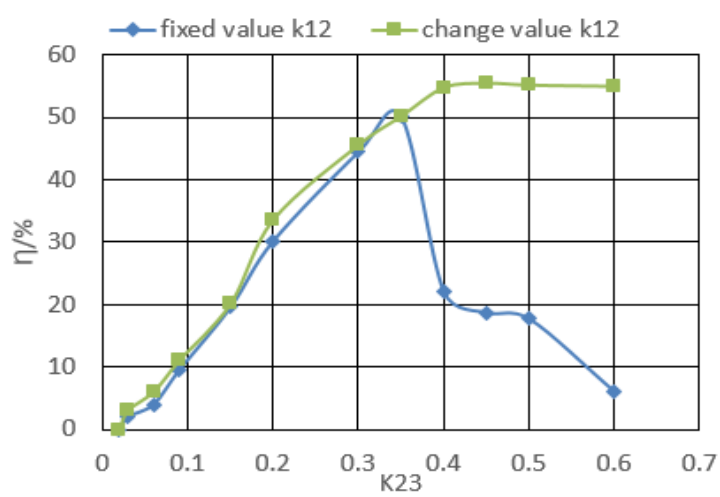

Figure 6. Curves of transmission efficiency

\subsection{Experiment verification}

Set up four coils of the wireless power transmission apparatus as shown in Figure 7. In the system, the transmission terminal and the receive terminal were integrated inspective for there was no influence on the experiment result according to the aforementioned analysis. This transmission circuit was charged by full bridge inversion with power MOS transistors IRF640 which were controlled by MOS driver UCC27424. And the receive coil was connected to diode rectification, including capacitor filtering. The load was high power LED. The WPT system was simplified so as to decrease the amount of work in the experiment, but it was enough for the accuracy of the result.

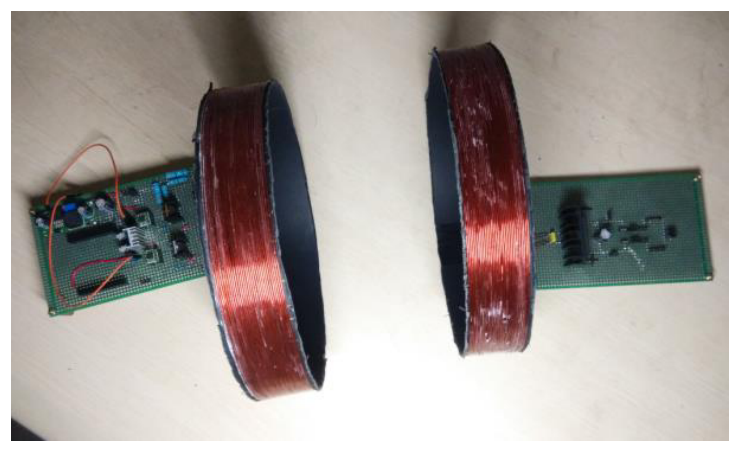

Figure 7. Test system of wireless power transmission

Keep other conditions such as circuit, coils and k34 unchanged and changing k12 and k23, we measured the input and output powers and calculated transfer efficiency. The result was shown in Figure 7 and Figure 8 .

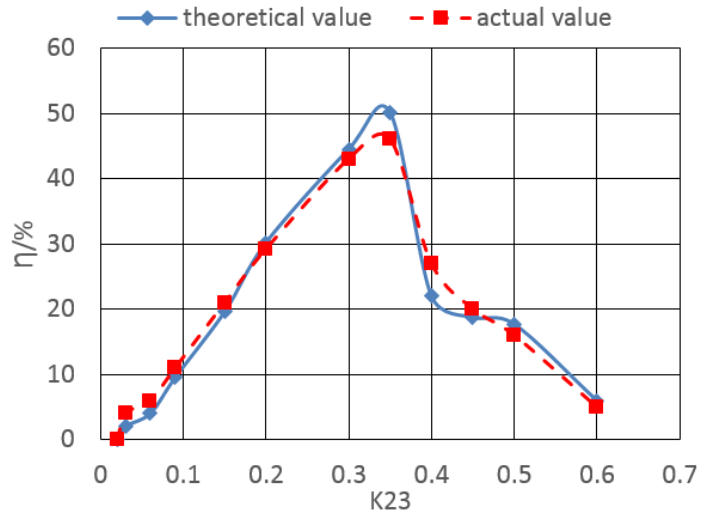

Figure 8. Transmission efficiency of experimental value compared with the theoretical value when $\mathrm{k} 12$ was fixed

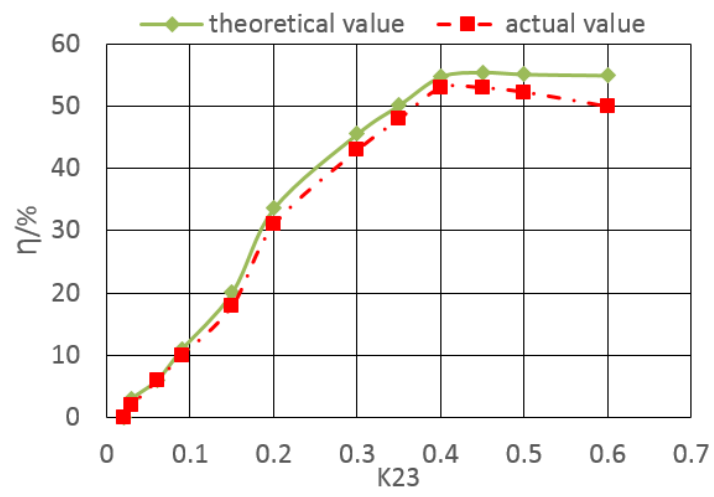

Figure 9. Transmission efficiency of experimental value compared with the theoretical value when $\mathrm{k} 12$ was changed 
From Figures 7 and 8, there was a little deviation between the experimental data and the simulation data. It was acceptable as the actual system stray inductance and stray capacitance existed, and taking into account the non-standard coil winding. Moreover, the resonant frequency of the parameter of the actual system was about $993 \mathrm{kHz}$ after constantly adjusting, while the actual use of resonant frequency was $1.01 \mathrm{MHz}$ in the simulation mentioned earlier. Take into account the tolerance limits, the results of the experiment will not cause much error.

\section{CONCLUSION}

This paper was mainly for portable electronic products, researching magnetic resonance coupling wireless power transmission system model, analyzing the model topology of the system deeply, discussing the relevant factors affecting the efficiency. And then, the relationship was obtained among the coil quality factor, coupling factor and the transmission efficiency, and optimized conditions in performance of this system which was analyzed, and the relational expression was given. Finally, verification through simulations and experiments show that it can effectively improve the system transmission efficiency for the wireless power transmission technology in portable electronic products by adjusting the coupling factor of the coils in transmitter terminal. It has laid a theoretical foundation.

\section{REFERENCES}

[1] SAMPLE A P, MEYER D A, \& SMITH J R. 2011. Analysis, experimental results, and range adaptation of magnetically coupled resonators for wireless power transfer. IEEE Transactions on Industrial Electronics, 58(2): 544-554.

[2] KURS A, KARALIS A. \& MOFFATT R, et al. 2007. Wireless power transfer via strongly coupled magnetic resonances. Scenic-express, 317(5834): 83-86.

[3] Jiaxun Wu, Junyong Wu. \& Yu Zhang, et al. 2012. Experimental Research on Wireless Power Transmission Based on Magnetic Coupling Resonant Theory. Modern Electric Power, 29(1): 24-28.

[4] Wenzhen Fu, Po Zhang. \& Dongyuan Qiu, et al. 2009. Maximum efficiency analysis and design of self-resonance coupling coils for wireless power transmission system. Proceedings of the CSEE, 29(18): 21-25.

[5] Yuan Zhai, Yue Sun. \& Xin Dai, et al. 2012. Modeling and analysis of magnetic resonance wireless power transmission systems. Proceedings of the CSEE, 32(12): 155-160.

[6] ISHIZAKI T. \& AWAI D F I. 2011. A novel concept for 2-dimensional free access wireless power transfer system using asymmetric coupling resonators with different sizes. IMWS, IEEE MTT-S International, 12(13): 243-246.
[7] DUONGTP, LEE J. W. 2011. Experimental results of high-efficiency resonant coupling wireless power transfer using a variable coupling method. IEEE Microwave and Wireless Components Letters, 21(8): 442-444.

[8] K. Scheurlen, A. Schnitzer. \& J. Krammer et al. 2014. Stellenwert. Der Galaktographie zur Abklärung pathologischer Sekretion in der komplementären Mammadiagnostik. Der Radiologe, 54(1).

[9] Teck C B, Imura T. \& Kato M, et al.2010. Basic study of improving efficiency of wireless power transfer via magnetic resonance coupling based on impedance matching. IEEE International Symposium on Industrial Electronics. Bari, Italy: IEEE, pp: 2011-2016.

[10] Seung-Hwan L. \& Lorenz R D. 2011. Development and validation of model for $95 \%$-efficiency $220-\mathrm{W}$ wireless power transfer over a 30 -cm air gap. IEEE Trans. on Industry Applications, 47(6):2495-2504.

[11]Y.-H. Kim, S.-Y. Kang. \& M.-L. Lee, et al. 2009. Optimization of wireless power transmission through resonant coupling. Proceedings of the Compatability and Power Electronics, (5): 426-431.

[12]Chunbo Zhu, Kai Liu, Chunlai Yu, Rui Ma. \& Hexiao Cheng. 2008. Simulation and experimental analysis on wireless energy transfer based on magnetic resonances. Harbin: IEEE Vehicle Power and Propulsion Conference, $\mathrm{pp}: 1-4$.

[13] Sunkyu K, Myunghoi K. \& Kyoungchoul K, et al. 2011. Analytical expressions for maximum transferred power in wireless power transfer systems. IEEE International Symposium on Electromagnetic Compatibility. Long Beach, USA: IEEE, pp: 379-383.

[14]Gabriele Grandi, Marian K·Kazimierczuk, Antonio Massarini. \& Ugo Reggiani. 1999. Stray capacitances of single-layer solenoid air-core inductors. IEEE Transactions on Industry Applications, 35(5): 1162-1166.

[15]Chunlai Yu, Rengui Lu, Yinhua Mao, Litao Ren. \& Chunbo Zhu. 2009. Research on the Model of Magnetic-Resonance Based Wireless Energy Transfer System. Dearborn, MI: Vehicle Power and Propulsion Conference, pp: 414-418.

[16]Thuc P D. \& Jong-Wook L. 2011. Experimental results of high-efficiency resonant coupling wireless power transfer using a variable coupling method. IEEE Microwave and Wireless Components Letters, 21(8): 442-444. 